\title{
An Efficient Truthful Double Spectrum Auction Design for Dynamic Spectrum Access
}

\author{
Enxin Yao, Luxi Lu and Wei Jiang ${ }^{\dagger}$ \\ School of Electronic Engineering and Computer Science, Peking University, CHINA \\ Email: †jiangwei@pku.edu.cn
}

\begin{abstract}
Spectrum auctions can motivate the legacy (primary) spectrum owners to lease their idle spectrum to other secondary spectrum users and eventually improve the spectrum utilization. Compared with traditional auctions, a significant difference of spectrum auctions is the spatial reusability, which means several buyers can use the same channel if they are far away from each other such that they will not interfere with each other. The challenge is how to exploit spatial reusability to improve spectrum utilization while keeping the auctions economicrobust (truthful in particular). In this paper, we propose TDSA, an efficient truthful double spectrum auction design to solve this problem, which has a novel virtual group bidding mechanism to improve spectrum utilization as well as a unique pricing strategy to guarantee truthfulness. We prove that our proposal is truthful. Experimental results show that our proposal can efficiently exploit spatial reusability of spectrum to achieve high spectrum utilization in most cases.
\end{abstract}

\section{INTRODUCTION}

The traditional exclusive licensing spectrum policy effectively protects the users from interfering with each other. However, this policy leads to the low efficiency of spectrum utilization. To solve this problem, DSA (Dynamic Spectrum Access), which allows secondary wireless networks to dynamically access the licensed bands from legacy spectrum holders has been focused recently [1].

Secondary spectrum auction is a promising solution for DSA. Compared with opportunistic spectrum access, auctions can motivate the licensed spectrum holders to lease their idle channels by market force. In fact, the governmental authorities such as FCC have already began to allocate the spectrum licenses by auctions since 1993 [2]. However, secondary spectrum auctions have different characteristics from traditional spectrum license auctions. Former efforts on auction-based dynamic spectrum access includes [3],[4],[5],[6].

Market manipulation could be quite detrimental to the fairness and efficiency of auctions. Truthfulness is quite helpful to prevent market manipulation. It also helps the buyers and sellers to avoid the expensive overhead to pick out their best strategies. Truthfulness (or strategy-proofness) means that no buyer or seller can improve its own utility by bidding untruthfully. [7] proposed an second-price auction for dynamic spectrum allocation where users have to pay for the his/her own communication time.[8] studied the truthful auctions where secondary wireless service providers have demands for fixed locations (cells). Vickrey-Clarke-Groves (VCG)[2] mechanism was used to maximum expected revenue while enforcing truth-

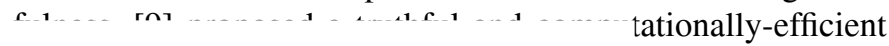

spectrum auction to support an eBay-like dynamic spectrum market. [10], [11] focus on truthful online auction where buyer's requests have different arrival time and departure time. In [12], a framework named TRUST is proposed for truthful spectrum auctions in double-sided markets, where are both multiple buyers and multiple sellers. It is based on McAfee [2] design and a pre-grouping mechanism for spectrum reuse which inspires our works in this paper. TRUST mainly focus on how to keep the double spectrum auction truthful when spectrum can be spatially reused. How to make more efficient usage of spatial reuse to improve the spectrum utilization is a problem worth exploring.

In this paper, we propose TDSA, an efficient truthful double spectrum auction design. With a novel group bidding mechanism and a unique pricing strategy, TDSA improves the spectrum utilization significantly while keeping truthfulness.

TDSA has made two contributions:

(1) With a novel virtual group bidding mechanism, TDSA can efficiently allocate and reuse the spectrum to improve spectrum utilization. We show the advantage by simulations.

(2) With a unique pricing strategy, TDSA keeps truthfulness as well as other economic properties which make TDSA an economic-robust auction design. We prove that TDSA has economic properties including truthfulness, individual rationality and ex-post budget balance.

\section{SySTEM MODEL}

We consider the scenario where the spatial coverage of sellers is much larger than that of buyers. This is practical because most primary spectrum licenses cover large regions. Assume there are $M$ large primary spectrum license holders want to lease out their temporary use right of spectrum channels to $N$ small local wireless networks. Therefore, the channels supplied by sellers can be shared by multiple non-conflicting buyers. To make this problem tractable, we assume each seller supplies only one channel. The channels from different sellers have uniform characteristics. Each buyer demands one channel.

A sealed auction is run by an auctioneer. When auction begins, sellers seal their quotations $Q_{m}, m \in\{1,2, \cdots, M\}$ in envelops and hand them to the auctioneer. At the same time, buyers seal their bids $B_{n}, n \in\{1,2, \cdots, N\}$ and hand them to the auctioneer. Then, the auctioneer opens up all these 
envelops and announces the final results, including the final spectrum allocation results and the clearing price $P_{m}^{s} / P_{n}^{b}$ for winning sellers / buyers.

\section{TDSA DETAILS}

In this section, we describe TDSA in details. To enable spatial reuse, TDSA first put the buyers into several groups where the members in the same group will not interfere with each other. Every group can be regarded as a super buyer. TDSA uses virtual group bidding mechanism to generate group bids for every group. Then, some sellers and buyer groups are selected according to McAfee rules. However, a selected buyer group does not necessarily means all the members in that group will win the auction. Some of the buyers in winning groups will be kicked out if their bids are too low. This washing out mechanism is critical to keep truthfulness and personal rationality in TDSA. After washing out, the clearing price for a buyer group is eventually shared by the remaining buyers within this group. TDSA consists of five steps.

\section{Step I: Buyer Group Formation}

The buyer group formation is performed by auctioneer with a bid-independent algorithm. The bid-independent group forming is quite helpful to keep truthfulness, which has already been shown in [12]. The buyer's interference condition is modeled as conflict graph. The group formation problem is equivalent to finding the independent sets of nodes in the conflict graph, which has been proved to be a NP-hard problem. We adopt existing approximate grouping algorithms in [13], [14] and denote the groups as $G_{i}, i \in\{1,2, \cdots, T\}$.

\section{Step II: Virtual Group Bidding}

Assume buyer $n$ with bid $B_{n}$ belongs to group $G_{i}$. The set of buyers (including buyer $n$ himself) whose bids are no less than $B_{n}$ is a virtual group. Assume there are $R$ members in group $G_{i}$, the virtual groups can be denoted as $V G_{j}, j \in\{1,2, \cdots, R\}$, according to their size. Fig. 1 shows the virtual groups.

Then, we generate virtual group bid for every virtual groups. Assume bids in group $G_{i}$ are sorted as $B O: B_{1} \geq B_{2} \geq$ $\cdots \geq B_{R}$. The virtual group bid $B_{j}^{v}$ for virtual group $V G_{j}$ can be described as follows:

$$
B_{j}^{v}=B_{j} \times j
$$

Finally, the group bid $G B i d_{i}$ for group $G_{i}$ is the highest virtual group bid. We describe group bid $\mathrm{GBid}_{i}$ as follows:

$$
\text { GBid }_{i}=\max _{j \in\{1,2, \cdots, R\}}\left(B_{j}^{v}\right)
$$

The detailed procedure is shown in Algorithm 1.

\section{Step III: Preliminary Winner Determination}

TDSA selects potential winners according to McAfee rules. The quotations of sellers are sorted in non-descending order and the group bids of buyers are sorted in non-ascending order.

$$
\begin{aligned}
& O^{\prime}: Q_{1} \leq Q_{2} \leq \cdots \leq Q_{M} \\
& O^{\prime \prime}: G B i d_{1} \geq G B i d_{2} \geq \cdots \geq \text { GBid }_{T}
\end{aligned}
$$
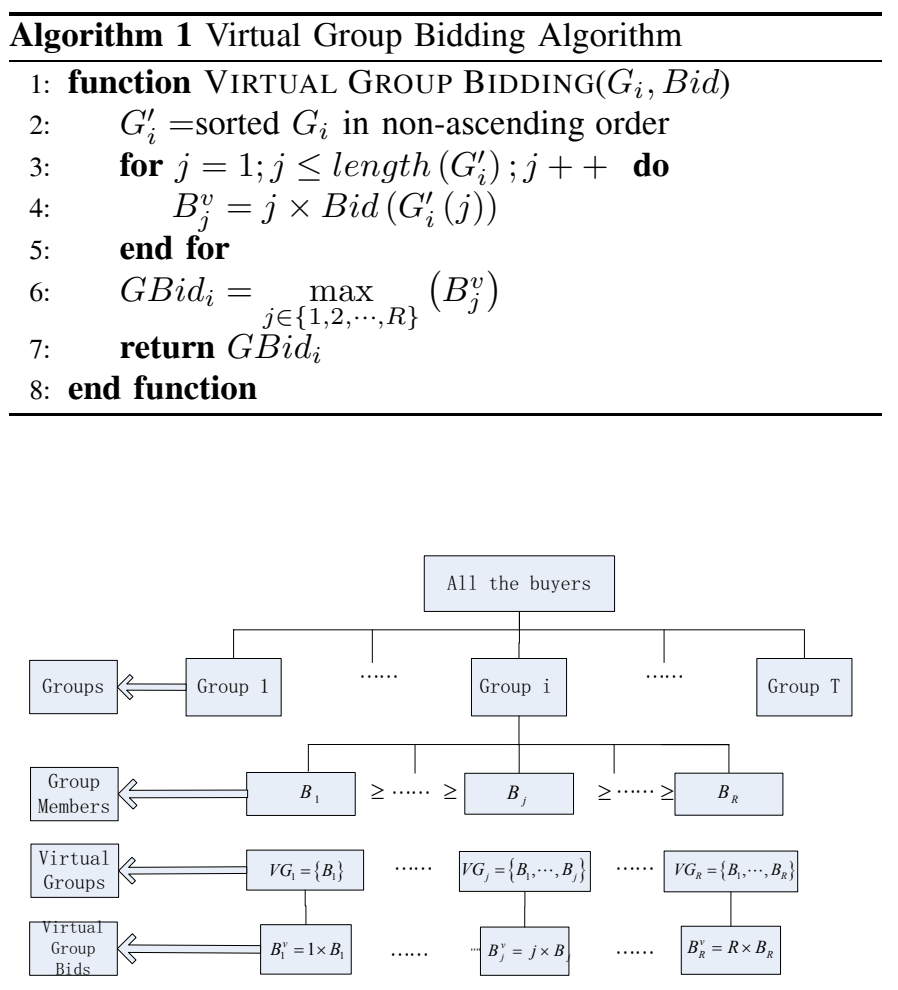

Fig. 1. Virtual Groups and Virtual Group Bids.

Define last profitable pair as follows:

$$
k=\underset{l \leq \min \{T, M\}}{\arg \max }\left\{\text { GBid }_{l} \geq Q_{l} \text { and } \text { GBid }_{l} \neq \text { GBid }_{l-1}\right\}
$$

The preliminary auction winners are top $k-1$ sellers in $O^{\prime}$ and top $k-1$ buyer groups in $O^{\prime \prime}$. The income for winning sellers is the $k_{t h}$ seller's quotation (i.e. $P^{s}=Q_{k}$ ). The price for winning buyer groups is the $k_{t h}$ buyer group's bid (i.e. $P G^{b}=G B i d_{k}$ ).

Note that the special condition $\mathrm{GBid}_{l} \neq G_{B i d_{l-1}}$ in (3) is necessary to keep TDSA truthful.

\section{Step IV: Washing Out}

In TDSA, the bids of some members in selected buyer groups are too low to afford the price. Consequently, washing out is carried out in each winning buyer group to delete these buyers. The remaining buyers are the final winners of the auction. The procedure of washing out is as follows: first, we sort the buyers in group $G_{i}$ according to their bids in non-ascending order, $B O: B_{1} \geq B_{2} \geq \cdots \geq B_{R}$. Then, we begin to wash out buyers one by one from $B_{R}$ to $B_{1}$, until we find a virtual group $\left\{B_{1}, B_{2}, \cdots, B_{c}\right\}$ whose virtual bid is larger than the price for winning buyer groups. (i.e. $B_{c}^{v}=B_{c} \times c>P G^{b}$ ). The detailed procedure is shown in Algorithm 2.

\section{Step V: Final Pricing}

TDSA pays each seller who has sold its channel by $P^{s}$, which is determined by the $k_{t h}$ seller's quotation in the sorting operation. For buyers, the price for the winning buyer groups is shared by the remaining members in the group after washing out. Assume buyer $n$ remains in a winning group $G_{i}$, there are 


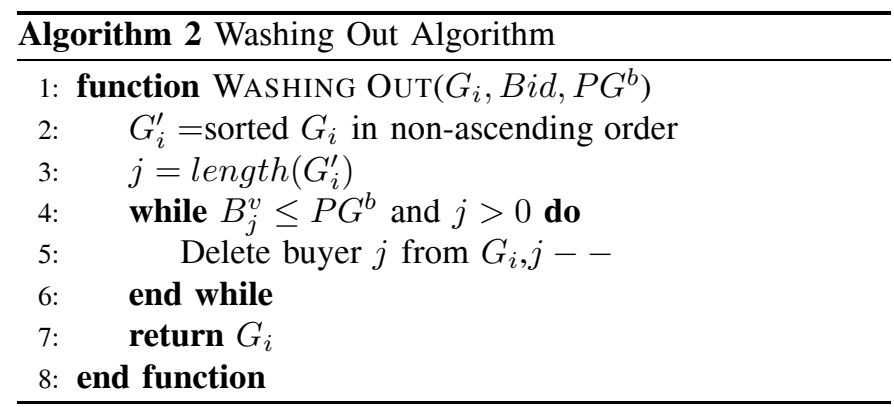

$H_{c}^{i}$ members left after washing out in group $G_{i}$. The clearing price for buyer $n$ is described as follows:

$$
P_{n}^{b}=\frac{P G^{b}}{H_{c}^{i}}
$$

\section{The AnAlysis of TDSA}

TDSA achieves high spectrum utilization by adopting a novel virtual group bidding mechanism. In this section, we compare this virtual group bidding mechanism with other group bidding mechanism.

The closed-form expression of expected value of group bid in TDSA is hard to derive, we calculate it by Monte-Carlo method. The results is shown in Fig.2(a). It is shown that the expected value of group bid in TDSA is almost proportional to the size of the group.

For former truthful auction design TRUST [12], group bid is generated by the lowest bid times the number of buyers in the group to guarantee truthfulness. Assume there are $R$ buyers in group $G_{i}$ and their bids are uniformly distributed between $[0, a]$. The expected value of $G B i d_{i}$ in TRUST is as follows:

$$
E\left(\text { GBid }_{i}\right)=R \int_{0}^{a} R\left(1-\frac{x}{a}\right)^{R-1} \frac{1}{a} x d x=\frac{a R}{R+1}
$$

When the number of buyers in the group increase, we have $\lim _{R \rightarrow+\infty} E\left(G B i d_{i}\right)=a$. This equation means that even if the group is huge, the mean value of group bid is still low, because the lowest bidding buyer hold the group back. Consequently, the large groups do not have advantage versus small groups. The spectrum are probably allocated to small groups instead of large groups. This will lead to low spectrum utilization.

Note that if the group bids are generated by the sum of the bids in the group, that would be very helpful for the auctioneer to pick out the most valuable groups. On the other hand, if the group bids are generated according to their size, that would help the auctioneer to increase the spectrum utilization. These two kinds of group bids seem to be very promising. Unfortunately, we can not find corresponding pricing strategy to keep the auction design truthful.

Fig.2(b) shows the group ranking results with different kinds of group bids. It is shown that the group ranking results in TDSA is highly relevant to the size of the group. The correlation coefficients of group ranking results are provided in Tab.1, averaged by the correlation coefficients in 100000 independent experiments. From Fig.2(b) and Tab.1, we can

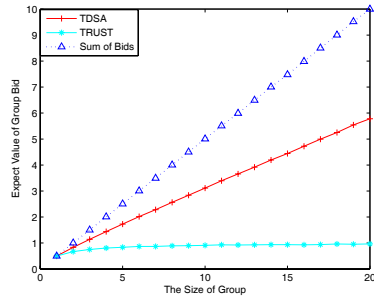

(a) The expected value of group bid by TDSA versus TRUST.

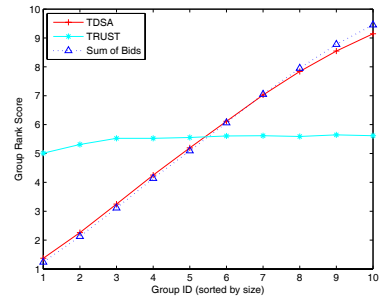

(b) Group ranking with different group bids.
Fig. 2. The results are averaged over 1000 times. The Bids of buyers are uniformly distributed between $[0,1]$.

TABLE I

CORRELATION COEFFICIENTS OF GROUP RANKING RESULTS FOR FOUR DIFFERENT GROUP BIDDING MECHANISMS

\begin{tabular}{c|c|c|c|c}
\hline Correlate & TDSA & TRUST & Sum of Bids & Group Size \\
\hline TDSA & 1 & 0.3212 & 0.9879 & 0.9636 \\
\hline TRUST & 0.3212 & 1 & 0.3091 & 0.3576 \\
\hline Sum of Bids & 0.9879 & 0.3091 & 1 & 0.9758 \\
\hline Group Size & 0.9636 & 0.3576 & 0.9758 & 1 \\
\hline
\end{tabular}

find out that the group ranking results in TDSA is quite relevant to the size of groups. This characteristic is quite helpful for the auctioneer to pick out the large groups and eventually improve the spectrum utilization.

Another characteristic also helps TDSA to increase spectrum utilization. It is easy to show that the group bid in TDSA is always no less than the group bid in TRUST for the same group. Higher group bids enable more buyer groups to afford the spectrum and increase the number of traded channels.

\section{PROOF OF ECONOMIC-PROPERTIES}

In this section, we prove that although every participants can manipulate their bids to change the auction results in TDSA, they do not have the incentive to do this. In other words, TDSA is truthful. We also prove TDSA achieve other economic-properties including individual rationality and expost budget balance. These properties make TDSA economicrobust and attractive to participants.

Theorem 1 (TDSA is truthful for buyers): In TDSA, no buyer can improve its own utility by bidding untruthfully.

In order to prove TDSA is truthful for buyers, we need to prove the following two lemmas at first.

Lemma 1: Assume buyer $n$ in group $G_{i}$ raise its bid while others bids keep unchanged. All the virtual bids and the group bid of group $G_{i}$ will be no less than before.

\section{Proof of Lemma 1:}

It is easy to show that when buyer $n$ raise its bid as $B_{n}^{\prime} \geq$ $B_{n}$, the lowest bid in each virtual group is no less than before (i.e. $B_{S(y)}^{\prime} \geq B_{y}, y \in\{1,2, \cdots R\}$ ). Consequently, the virtual group bids will be no less than before (i.e. $B_{y}^{\prime v}=B_{S(y)}^{\prime} \times y \geq$ $\left.B_{y} \times y=B_{y}^{v}, y \in\{1,2, \cdots R\}\right)$. Because the group bid is the 
TABLE II

X MEANS BUYER FAILS THE AUCTION WHILE $\sqrt{ }$ MEANS BUYER WINS

\begin{tabular}{c|c|c|c|c}
\hline case & $\mathbf{1}$ & $\mathbf{2}$ & $\mathbf{3}$ & $\mathbf{4}$ \\
\hline The buyer lies & $\mathrm{X}$ & $\mathrm{X}$ & $\sqrt{ }$ & $\sqrt{ }$ \\
\hline The buyer bids truthfully & $\mathrm{X}$ & $\sqrt{ }$ & $\sqrt{ }$ & $\mathrm{X}$ \\
\hline
\end{tabular}

highest virtual group bid, the group bid is no less than before either.

Lemma 2: In TDSA, if buyer $n$ wins the auction by bidding $B_{n}$, given other bids and quotations keep unchanged, buyer $n$ will still win by bidding $B_{n}^{\prime}>B_{n}$ and the charge for buyer $n$ will not change.

\section{Proof of Lemma 2:}

Assume the winning buyer $n$ belongs to buyer group $G_{i}$. To prove buyer $n$ will win when bids $B_{n}^{\prime}$, we have to prove group $G_{i}$ will win at first.

When buyer $n$ bids $B_{n}^{\prime}>B_{n}$, according to lemma 1, we have $G B i d_{i}^{\prime} \geq G B i d_{i}>P G^{b}$. Because both $G B i d_{i}^{\prime}$ and $G_{B i d}$ are higher than $P G^{b}$, we have $P G^{\prime b}=P G^{b}$. Group $G_{i}$ will still win and the price for group $G_{i}$ keep unchanged.

When buyer $n$ bids $B_{n}$, assume the lowest bid of the remaining buyers in $G_{i}$ after washing out is $B_{c}$, we have $B_{n}^{\prime}>B_{n} \geq B_{c}$. Because both $B_{n}^{\prime}$ and $B_{n}$ are higher than $B_{c}$, buyer $c$ will still be the lowest bid of remaining buyers when buyer $n$ bids $B_{n}^{\prime}$. The number of remaining buyers after washing out is the same as before. The price charged to buyer $n$ will not change.

Now we begin to prove theorem 1 with lemma 1, 2 .

\section{Proof of Theorem 1:}

We prove theorem 1 by discussing four cases for an arbitary buyer $n$, as shown in Tab.2.

$\diamond$ Case 1: For both sides, buyer fails in the auction, the utilities for both sides are zero.

$\diamond$ Case 2: If buyer wins by bidding truthfully, the utility will be no less than zero (see theorem 3 ). When buyer lies and loses, the utility will be zero. The buyer cannot obtain higher utility by bidding untruthfully.

$\diamond$ Case 3: According to lemma 2, the price for buyer will not change. The utility will not change either.

$\diamond$ Case 4: Assume buyer $n$ belongs to group $G_{i}$. When buyer $n$ bids truthfully $B_{n}=V_{n}$ ( $V_{n}$ is buyer's valuation of the channel) and loses the auction, we have $V_{n}=B_{n} \leq \frac{P G^{b}}{H_{n}} . H_{n}$ is the number of buyers whose bids are no less than $B_{n}$ in group $G_{i}$. When buyer $n$ lies and wins, according to the contrapositive of lemma 2 , we have $B_{n}^{\prime}>B_{n}=V_{n}$. According to lemma 1, we have $G B i d_{i}^{\prime} \geq G B i d_{i}$. As other bids / quotations keep unchanged, the price for winning buyer groups will be no less than before (i.e. $P G^{\prime b} \geq P G^{b}$ ).

We consider two situations as follows:

First, assume buyer $n$ is the lowest buyer after washing out when buyer $n$ lies and there are $H_{n}^{\prime}$ remaining buyers. Because $B_{n}^{\prime}>B_{n}$, we have $H_{n}^{\prime} \leq H_{n}$. For this situation, the utility for buyer $n$ is $U_{n}^{b}=V_{n}-\frac{P G^{\prime b}}{H_{n}^{\prime}} \leq \frac{P G^{b}}{H_{n}}-\frac{P G^{\prime b}}{H_{n}^{\prime}} \leq 0$.

Second, assume buyer $c$ instead of buyer $n$ is the lowest buyer after washing out when buyer $n$ lies. We have $B_{n}^{\prime} \geq B_{c}$ because buyer $n$ wins. Assume there are $H_{c} / H_{c}^{\prime}$ buyers whose bids are no less than buyer $c$ when buyer $n$ bids $B_{n} / B_{n}^{\prime}$. We have $H_{c}^{\prime} \leq H_{c}+1$ because only buyer $n$ raises its bid. Because buyer $n$ loses the auction when it bids truthfully, we can prove $B_{n}<B_{c}$ by reduction to absurdity method. Thus, we have $H_{n} \geq H_{c}+1 \geq H_{c}^{\prime}$. The utility for buyer $n$ is $U_{n}^{b}=V_{n}-\frac{P G^{\prime b}}{H_{c}^{\prime}} \leq \frac{P G^{b}}{H_{n}}-\frac{P G^{\prime b}}{H_{c}^{\prime}} \leq 0$.

From the analysis of four cases, we have proved that no buyer can improve its utility by bidding untruthfully. Consequently, TDSA is truthful for buyers.

Theorem 2 (TDSA is truthful for sellers): In TDSA, no seller can improve its own utility by bidding untruthfully.

The proof of theorem 2 is quite similar to the proof of theorem 1. To keep concise, we omit this proof here.

Theorem 3 (TDSA is individual rational): In TDSA, no winning seller is paid less than its quotation and no winning buyer pays more than its bid.

\section{Proof of Theorem 3:}

For the lost sellers and buyers, the clearing price is zero. They are individual rational.

For winning sellers, their quotations $Q_{m}$ are no more than their pay $Q_{m} \leq P^{s}=Q_{k}$ because they rank higher than $Q_{k}$ in the sorting operation.

For winning buyers, assume buyer $n$ belongs to group $G_{i}$. According to washing out rules, the virtual group bid $B_{c}^{v}$ for the remaining buyers after washing out is higher than the clearing price for the buyer group $P G^{b}$ (i.e. $B_{c}^{v}=B_{c} \times c>P G^{b}$ ). The clearing price $P_{n}^{b}$ for the buyer $n$ is no more than its bid $B_{n}$. (i.e. $P_{n}^{b}=\frac{P G^{b}}{c}<B_{c} \leq B_{n}$ ).

Theorem 4 (TDSA is ex-post budget balanced): In TDSA, the auctioneer's profit is always no less than 0 .

\section{Proof of Theorem 4:}

In TDSA, the auctioneer collects money from the top $k-1$ buyer groups by $P G^{b}=G B i d_{k}$ and pay money to the top $k-1$ sellers by $P^{s}=Q_{k}$. The utility of auctioneer is as follows:

$$
U^{a}=(k-1) \times\left(\text { BBid }_{k}-Q_{k}\right) \geq 0
$$

\section{EXPERIMENTAL RESULTS}

In this section, we use experimental results to show the high efficiency of TDSA on spectrum utilization as well as other metrics. We compare our proposal with the famous truthful double spectrum auction framework: TRUST[12]. It is a fair compare because we have proved that TDSA achieves the same economic properties as TRUST in previous section.

Assume there are 50 buyers randomly distributed in an area of $500 \times 500$ and 10 channels to be sold. If the distance of two buyers is less than 100 , they will interfere with each other. The bids of buyers / quotations of sellers are uniformly distributed between $[0,2] /[0,6]$.

Both TDSA and TRUST can cooperate with different group formation algorithms. We try different polynomial-time maximal independent set algorithms of conflict graph such as Max-IS (Using minimum maximum independent set of node's 


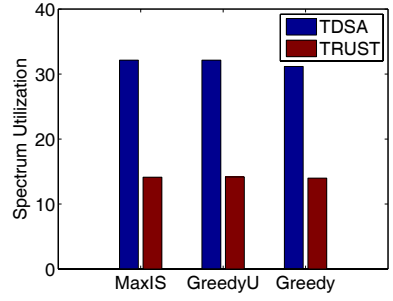

(a) Spectrum Utilization

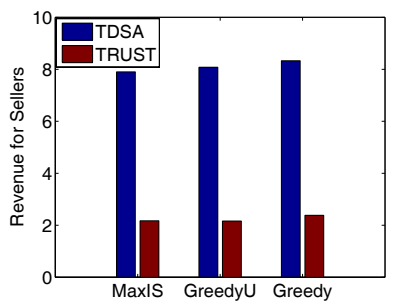

(c) Revenue for Sellers

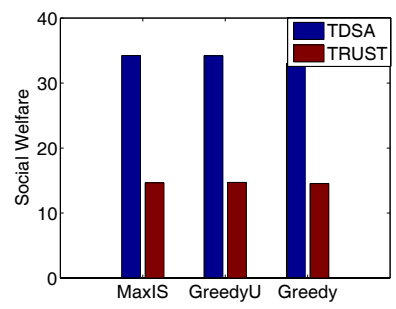

(b) Social Welfare

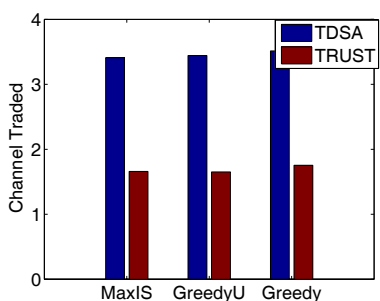

(d) Amount of Traded Channels

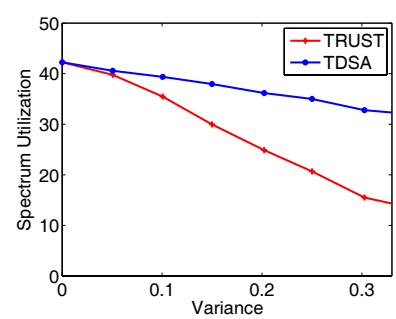

(a) Spectrum Utilization

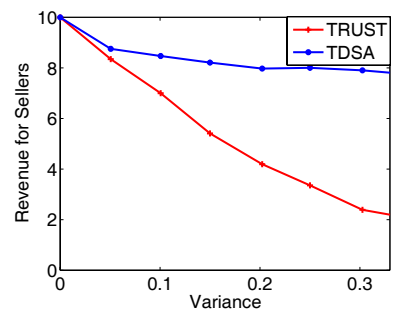

(c) Revenue for Sellers

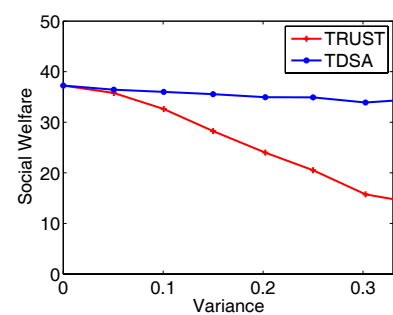

(b) Social Welfare

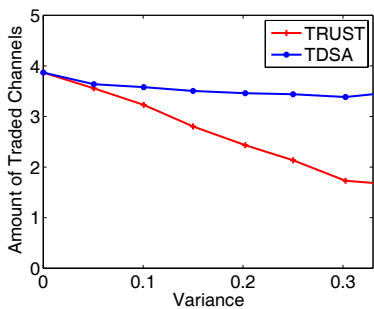

(d) Amount of Traded Channels

Fig. 3. The performance of TDSA and TRUST with Max-IS, Greedy-U and Greedy. The results are averaged over 1000 times.

neighborhood) [13], Greedy-U and Greedy (Using node's progressive/static degree)[14]. Different metrics including spectrum utilization, social welfare [12], revenue for the sellers [8] and the amount of traded channels are used to evaluate the performance. Fig. 3 shows TDSA outperforms former truthful auction design TRUST on all these four metrics which confirms our analysis in part IV.

However, the performance of TDSA is not always so much better than TRUST as shown in Fig.3. The performance is highly relevant to the distribution of buyer's bids, especially the variance. Fig. 4 shows that when buyers have the same bids, the performance of TRUST and TDSA is just the same. However, as the variance of buyer's bids increase, the high efficiency of TDSA begin to show up.

\section{CONCLUSION}

In this paper, we propose an efficient truthful double spectrum auction design for dynamic spectrum access. With a novel virtual group bidding mechanism, our proposal can efficiently exploit the spatial reusability of spectrum to increase the spectrum utilization in auction-based dynamic spectrum access. We prove that our proposal can achieve economic properties including individual rationality, ex-post budget balance and truthfulness to make it an economic-robust auction. Simulation results show our proposal has high efficiency on spectrum utilization as well as other metrics. It is generally more efficient compared with former design, especially when buyer's bids are variable.

\section{ACKNOWLEDGMENT}

Our works are supported by NSFC Grant No.60972008 and NDFC Grant No.9140A220310.
Fig. 4. Performance with different variances of buyer's bids. The bids are distributed between $[0,2]$ with the mean value of 1 .

\section{REFERENCES}

[1] Ian F. Akyildiz, Won-Yeol Lee, Mehmet C. Vuran and Shantidev Mohanty, NeXt generation/dynamic spectrum access/cognitive radio wireless networks: A survey, Computer Networks, 50, 2127-2159, 2006.

[2] Paul Milgrom. Putting Auction Theory to Work, Cambridge University Press, 2004. ISBN: 0521536723.

[3] J. Huang, R. Berry, and M. Honig, Auction-based Spectrum Sharing, ACM/Kluwer MONET special issue on WiOpt04, 2004.

[4] Zhu Ji, K.J.Ray Liu, Dynamic Spectrum Sharing: A Game Theoretical Overview, IEEE Communication Magazine, vol. 45, issue 5, May. 2007, pp. 88-94.

[5] Sengupta, Shamik, Chatterjee and Mainak, Sequential and Concurrent Auction Mechanisms for Dynamic Spectrum Access, IEEE CrownCom 2007, pp. 448-455.

[6] L. Gao, Y. Xu, Xinbing Wang, MAP: Multi-Auctioneer Progressive Auction in Dynamic Spectrum Access IEEE Transactions on Mobile Computing, 2010.

[7] A. T. Hoang and Y.-C Liang, Dynamic Spectrum Allocation with Second-Price Auctions: When Time is Money, IEEE CrownCom 2008.

[8] J. Jia, Q. Zhang, Qin. Zhang, M. Liu, Revenue Generation for Truthful Spectrum Auction in Dynamic Spectrum Access, Proc. of MobiHoc, 2009.

[9] Z. Xia, S. Gandhi , S. Suri, H. Zheng, ebay in the sky: Strategy-proof wireless spectrum auctions, Proc. of MobiCom, 2008.

[10] S. Wang, P. Xu, X. Xu, S. Tang, X. Li, X. Liu, TODA: Truthful Online Double Auction for Spectrum Allocation in Wireless Networks, Proc. of DySPAN, 2010.

[11] S. Sodagari, A. Attar, S.G. Bil'en, Implications of Dynamic Spectrum Access on the Efaciency of Primary Wireless Market, Proc. of DySPAN, 2010.

[12] Z. Xia, H. Zheng, TRUST: A General Framework for Truthful Double Spectrum Auctions, Proc. of Infocom, 2009.

[13] A. Subramanian, H. Gupta, S. Das, M. Buddhikot, Fast spectrum allocation in coordinated dynamic spectrum access based cellular networks, Proc. of DySPAN, 2007.

[14] S. Ramanthan. A uniCed framework and algorithm for channel assignment in wireless networks, Wireless Networks, 5(2): 81-94, 1999. 